\title{
Conservation of single amino-acid polymorphisms in Plasmodium falciparum erythrocyte membrane protein 1 and association with severe pathophysiology
}

\author{
Daniel Zinder $^{\mathbf{a}^{*}}$, Mary M. Rorick ${ }^{\mathbf{a}}$, Kathryn E. Tiedje ${ }^{\mathbf{b c}}$, Shazia Ruybal-Pesántez ${ }^{\mathbf{b c}}$, Karen. P. Day ${ }^{\mathbf{b c}}$, \\ Mercedes Pascual $^{\text {ad }}$ \\ a University of Chicago, Ecology and Evolution, 1101 E 57th Street, Chicago, IL 60637, USA \\ b School of Biosciences, The University of Melbourne, Melbourne, AU \\ c Department of Microbiology, New York University, New York, USA \\ d Santa Fe Institute, Santa-Fe, NM 87501, USA \\ * corresponding author
}

Corresponding author:

E-mail: dzinder@uchicago.edu

Mail: University of Chicago, Ecology \& Evolution, 1101 E 57th Street, Chicago, IL 60637, USA Phone:

lab - (773) 795-2354

\begin{abstract}
Plasmodium falciparum erythrocyte membrane protein 1 (PfEMP1) is a parasite protein encoded by a multigene family known as var. Expressed on the surface of infected red blood cells, PfEMP1 plays a central role in parasite virulence. The $D B L \alpha$ domain of PfEMPl contains short sequence motifs termed homology blocks. Variation within homology blocks, at the level of single amino-acid modifications, has not been considered before in association with severe disease. Here we identify a total of 2701 amino-acid polymorphisms within $D B L \alpha$ homology blocks, the majority of which are shared between two geographically distant study populations in existing transcription data from Kenya and in a new genomic dataset sampled in Ghana. Parasitemia levels and the transcription levels of specific polymorphisms are as predictive of severe disease $(\mathrm{AUC}=0.83$ ) and of the degree of rosetting (forecast skill $\mathrm{SS}=0.45)$ as the transcription of classic var groups. 11 newly categorized polymorphisms were strongly correlated with grpA var gene expression ( $\mathrm{SS}=0.93$ ) and a different set of 16 polymorphisms was associated with the $H 3$ subset $(\mathrm{SS}=0.20$ ). These associations provide the basis for a novel method of relating pathophysiology to parasite gene expression levels - one that, being site-specific, has more molecular detail than previous models based on var groups or homology blocks. This newly described variation influences disease outcome, and can help develop anti-malarial intervention strategies such as vaccines that target severe disease. Further replication of this analysis in geographically disparate populations and for larger sample sizes can help improve the identification of the molecular causes of severe disease.
\end{abstract}

\section{INTRODUCTION}

The manifestation of Plasmodium falciparum infection is wildly variable. In highly endemic areas the vast majority of infections are asymptomatic and most cases of clinical malaria are mild. Only a small fraction of individuals with clinical malaria, predominantly infants, go on to develop life-threatening severe disease $(1,2)$. Severe cases represent less than one percent of infected individuals, but they are nevertheless the dominant cause of morbidity and mortality from this disease (3-5). Despite decades of intensive control efforts, there are approximately 212 million cases of malaria annually, and hundreds of thousands of deaths due to P. falciparum infection, the majority of which are children in Africa (6). The fact that most people in endemic regions rapidly develop immunity to complicated malaria within the first 
few years of life offers hope for the possibility of developing targeted vaccines to prevent severe disease (7).

In the bloodstream, P. falciparum infects red blood cells and then uses a diversity of human receptors to promote the adherence and sequestration of infected red blood cells (iRBCs) within various human tissues. Parasite density, the fraction of iRBCs, is associated with increased virulence, and $P$. falciparum' $s$ unique ability to readily invade mature RBCs contributes to its increased pathogenicity (8). While a high parasite burden is more common during severe disease, improved control of parasite density alone does not explain resistance to severe disease (9). iRBCs avoid clearance by the spleen by tethering to the linings of small blood vessels in various host tissues, and by binding uninfected cells - a phenomenon called rosetting. Massive iRBC sequestration in host microvasculature strongly contributes to virulence (10). Some severe complications of malaria are associated with parasite sequestration in particular tissues: e.g. in the microvasculature of the brain in the case of cerebral malaria, and the placenta in the case of pregnancy-associated malaria. Other severe pathophysiologies include extreme weakness, convulsions, renal failure, circulatory shock, low blood glucose levels, acidosis, respiratory distress, impaired consciousness and severe malarial anemia (11).

The adherence and sequestration of the parasite in particular tissue types, as well as the rosetting phenotype, are strongly associated with the expression of specific members of the var gene family (12, 13). The var genes encode Plasmodium falciparum erythrocyte membrane protein 1 (PfEMP1), and specific PfEMP1 types have been found to be preferentially expressed in particular host tissues including the brain and cardiac tissue of severely diseased infants and in infected placental tissue $(12,14-16)$. Located in multiple subtelomeric and central chromosomal regions, approximately 60 antigenically distinct PfEMP1 variants are encoded per parasite genome. PfEMP1 proteins are large (200-350 kDa) and come in a diversity of architectural types, which are defined by the presence and multiplicity of specific domains (17). The major architectural subtypes can be classified into three groups-A, B and C, and they are associated with distinct chromosomal locations, transcription direction, and semi-conserved upstream sequence (ups) tags (18-20). Because group A/B/C var genes are commonly classified by their ups tags, and because we do not have ups sequences for the var diversity considered here, we will use "grpA-like" to refer to a group of var gene sequences classified by an alternative network-based method previously established to be highly correlated with ups-based group A classification $(13,21)$.

Because of its important role in binding parasite-infected cells to host microvasculature, the extracellular domains of PfEMP1 are under strong natural selection for affinity to host endothelial receptors. Residing in high density on the outside of infected cells, these domains are also under strong natural selection to evade the adaptive immune system. The parasite employs an antigenic variation system whereby individual parasites only express a single var gene at a time, switching expression over the course of an infection $(8,22,23)$. The regulation of var gene expression is not yet fully elucidated, though it appears to centrally involve epigenetic machinery (24-26). As a probable outcome of immune evasion, var gene sequences are highly diverse both within individual parasite genomes and at the population level. Withindomain amino acid identity is less than $50 \%$, even within the same architectural type $(20,27)$. Although sterilizing immunity never develops against $P$. falciparum, the severity of disease is rapidly reduced with repeated infection $(7,28,29)$. A component of naturally acquired immunity to disease complications appears to involve an antibody response to PfEMP1 (30-32). As such, identifying conserved epitopes of PfEMP1 which are associated with severe disease is of major interest $(23,33,34)$. 
Detailed subtyping has enabled the identification of domains and domain cassettes which bind to specific endothelial tissue receptors and to placental tissue $(20,35-41)$. The head structure of the PfEMP1 protein contains two domains referred to as CIDR and DBL $\alpha$. Increased transcription of group A vars has been linked to rosetting and to a higher likelihood of severe disease $(21,42-45)$. This is possibly the result of the ability of head structure variants, which are associated with group A var types, to preferentially bind endothelial protein $\mathrm{C}$ receptor (EPCR) and mediate the formation of rosettes $(20,46)$. Sequence motifs, such as H3, have also been linked with the rosetting phenotype (47). In addition to the head structure, other domains of PfEMP1 have been implicated in pathology. These include the DBL $\beta$ in its binding of ICAM-1 in cerebral malaria and the binding of various domains of the var2csa in placental malaria (48, 49). In contrast with group A vars, the head domains in group B and C vars have been shown to preferentially bind to the host's CD36 receptor $(17,50)$. The transcripts of group B vars have been identified as more common in symptomatic infection of children with malaria (17).

Host genetics also play a central role in determining the severity of disease. The carrier status of traits such as $\mathrm{HbS}$ thalassemia, glucose-6-phosphate dehydrogenase deficiency, complement receptor (CR) 1 deficiency, sickle cell traits and duffy blood groups appears to confer a degree of protection against severe malaria in certain populations (51-56). In addition, in other populations, different ABO blood groups have been shown to reduce rosetting frequency, with group $\mathrm{O}$ being the most protective against severe disease (57). Host genetic variation conferring malaria resistance appears to generally be due to recent adaptation (5000-10,000years), vary considerably between human populations and be dependent on parasite genetics (58).

An important advance has been made with the discovery of short conserved sequence motifs or 'homology blocks' within the PfEMP1 coding sequence, many of which have unique cytoadhesion traits and have been implicated in severe disease. Some have been shown to have preferential expression in patients with severe disease symptoms (e.g., (13). Here we consider variation within homology blocks of the DBL $\alpha$ domain, its conservation across populations, and its relation to severe disease symptoms.

\section{METHODS}

\section{Study site and sampling of DBL $\alpha$ diversity for the genomic dataset}

The "genomic data" used in this study was collected in Bongo District (BD), located in the Upper East Region (UER) of Ghana. Details on the study design, study population and data collection procedures have been described previously (Ruybal-Pesántez et al. 2017). To summarize here: sampling was carried out in two sites ("catchment areas") of similar human population size, age structure and ethnic composition located $\sim 10-40 \mathrm{kms}$ apart. Vea/Gowrie was expected to possibly exhibit higher and/or less seasonal transmission than Soe because of its proximity to the Vea dam/irrigation area. The catchment areas were further divided into smaller villages: Vea, Gowrie, Soe Sanabisi and Soe Boko, with participants enrolled from "sections" within these villages (Vea: Gonga and Nayire; Gowrie: Nayire Kura and Tingre; Soe Sanabisi: Tindingo and Akulgoo; and Soe Boko: Tamolinga and Mission Area), meaning that the final dataset contains isolates from one of eight sub-populations in total. All individuals were surveyed in June 2012, which is near the end of the dry season when parasite population sizes and diversity are expected to be at their lowest. Methods related to the microscopy, msp2 PCR and the microsatellite PCR are described in detail in (Ruybal-Pesántez et al. 2017). Var DBL $\alpha$ tags were sequenced for 209 parasite positive samples. 


\section{Defining distinct var types:}

Throughout this study we focus on the genetic diversity within the var DBL $\alpha$ domain because it is the only domain found in nearly all var genes. It is also highly conserved relative to other regions of the var gene. For these reasons it is the generally accepted and most popular molecular marker of var gene diversity for field-based studies $(41,59-61)$. For the genetic data, the DBL $\alpha$ sequences were assigned to var types using a clustering algorithm in a manner consistent with the commonly used $96 \%$ nucleotide identity definition for field studies (62). Each var type cluster corresponds roughly to sequences with a $>97 \%$ amino acid sequence identity. This threshold is consistent with the majority of prior work defining distinct types within DBL $\alpha$ tag sequences because it ensures that each distinct sequence type is very likely to represent a naturally occurring distinct variant, and not merely the result of sequencing errors. Homology blocks and homology block's polymorphisms were identified within distinct var gene types.

\section{HB polymorphism identification}

We translated DNA sequences to AA sequences using the software program EMBOSS Transeq $(63,64)$. We excluded from the analysis sequences that had an unexpected reading frame, apparent frame shift substitutions or stop codons. Homology blocks (HBs) are conserved units of recombination that are present in var genes. Here we only consider those that occur within the DBL $\alpha$ tags of our datasets. HBs were identified using the VARDOM web server (65), with a gathering cut-off of 9.97 to define a match. HBs of a specific type were aligned and all the amino-acid variation in sites containing more than one amino-acid variation were catalogued (Fig. 1).

\section{Genomic samples}

Three genomic isolates were used as positive controls for our sequencing and analysis methods: 3D7, DD2, and HB3. These isolates have a known multiplicity of infection $(=1)$, and the number of var genes that exist per genome has been previously established. The sequence of each of the DBL $\alpha$ tag sequences within these genomes is also known with high accuracy. For these isolates we can distinguish sequencing errors from within-type variants, and therefore identify multiple var sequences of the same type.

\section{Transcription samples}

The expressed sequences and the clinical data for 250 isolates were obtained from the online supplementary information of (21). The Warimwe et al. study included children recruited between August 2003 and September 2007 from the Kilifi District Hospital, situated at the coast of Kenya. In their study DBL $\alpha$ sequence tags were amplified from parasite cDNA sampled from each of $112(44.8 \%)$ children with severe malaria, $105(42 \%)$ children with symptomatic non-severe malaria, and $33(13.2 \%)$ asymptomatic children.

Here, we divided the "expression dataset" of Warimwe et al. randomly into a validation dataset and a training dataset, containing $175(70 \%)$ and $75(30 \%)$ individuals, respectively. Training and normalization were performed using the training dataset expression levels only. Prediction is reported with respect to the validation dataset.

\section{Transformation of expression rates and rosetting level}

Prior to performing all linear and logistic regression analyses, the expression rates of particular var types (i.e., cys2, A-like, group 1, group 2, group 3, BS1/CP6 and H3sub var genes), of homology blocks (i.e. for all $29 \mathrm{HBs}$ ), and of homology block polymorphisms (i.e. 2148 polymorphisms in the Warimwe et al. 
dataset) were normalized and log transformed. Normalization was performed by dividing with the corresponding median expression rates in non-severe and asymptomatic cases in the training dataset. Prior to the $\log$ transform, extreme (i.e. zero and one) expression values were replaced with $\frac{1}{4 \cdot N_{\text {clones }}}$ and 1 $\frac{1}{4 \cdot N_{\text {clones }}}$, respectively. Rosetting and parasite density levels equal to zero were replaced with $\frac{1}{4 \cdot 200}$ and $\frac{1}{2}$, respectively, prior to the log transform. These types of replacements were also carried out by (45), and they are for numerical reasons and to account for the limited sensitivity of detection., .

\section{Predictive Ability}

Because of the large dimensionality of the data, and the large number of predictive variables in comparison to the number of study individuals $(\mathrm{p}>>\mathrm{n})$, the predictive ability of different models was evaluated using a separate dataset which was not included in data normalization or training. For binaryoutcomes, predictive ability was calculated using the 'area under the curve' (AUC) statistic. Given a list of disease state prediction scores generated by the model for each individual in the validation dataset, the AUC is the probability that a disease positive individual will get a higher score in comparison to a disease-free individual. For continuous outcomes, predictive ability was calculated using the forecast skill (SS) in comparison to the training dataset mean. This is equivalent to an $r^{2}$ statistic with the difference that the linear fit is created using the training dataset and the statistic is calculated for the validation dataset.

\section{Model Selection}

Model selection was performed using the 'Sparse Group LASSO' R package (66). Similar to the lasso method, this method minimizes a weighted sum of the model prediction error and the absolute value of the regression coefficients. As such it prefers sparse solutions. A stochastic implementation of this procedure is repeated several times, the model used includes only variables which appear in at least a certain percent of models, referred to as stable variables. A threshold of $90 \%$ of models was used for logistic models. A different threshold of $20 \%$ evaluated empirically was used for inclusion of variables in the linear prediction of rosetting.

The sparse group lasso method assigns a penalty to groups of coefficients together. Polymorphisms were assigned to groups based on belonging to the same site in the homology block sequence, the remaining variables were not assigned into groups. An equal weight was given to the lasso and group lasso penalties $(\alpha=0.5)$.

\section{False discovery rates}

False discovery rates (q-values) were calculated using the algorithm described in (67) R package 1.38.0. This method offers a more powerful way of correcting for multiple comparisons in relation to e.g. Bonferroni correction, and is expected to provide more robust estimates in comparison to BenjaminiHochberg (68). It uses an interpolation approach for estimating the excess number of $p$-values of a given value and thus the false discovery rate.

\section{RESULTS}

\section{Diversity of amino-acid polymorphisms in Ghana and in Kenya}

We measured the fraction of individuals, and the fraction of vars within each individual, in which specific single amino-acid polymorphisms within homology blocks were present. This was done in genomic data 
from the study population in the Bongo district of Ghana in 2014, and in expression data from the Warimwe et al. 2009 study in the Kalifi district of Kenya.

We identified a total of 2701 single amino acid polymorphisms (PMs) occurring at 316 different sequence positions (sites) (Fig. 2). 2098 (77.6\%) polymorphisms across 305 (97\%) sites were shared between the two study populations/dataset types. A total of 48 (1.8\%) PMs were only present in expression data from Kenya, whereas a higher total of 555 (20.5\%) PMs were only present in the genomic dataset from Ghana. The fraction of individuals with a given PM (the population-level frequency) was highly correlated between the two studies (Spearman's rank $\rho=0.96, p=0$ ) (Fig. 2), indicating that the population-level frequency of PMs is actively maintained by evolution across vast geographic ranges (the two study sites are $5000 \mathrm{~km}$ apart), even in the diverse parasite populations of sub-Saharan Africa.

On average of $1030 \pm 290$ (mean \pm std) PMs were identified per individual from the genomic data from Ghana, while roughly half (550 $\pm 290 \mathrm{PMs}$ ) were identified per individual from the transcription data from Kenya. Because there are multiple var copies per parasite genome, and often multiple parasite genomes within a given individual host, PMs can occur multiple times within an individual. Therefore, PMs have an individual-level frequency in addition to a population-level frequency. The individual-level frequency of a given PM was correlated among the individuals within a population (average Spearman's $\langle\rho>=0.61 \pm 0.1$ in Kenya, $\langle\rho>=0.78 \pm 0.1$ in Ghana) and between the two populations $(<\rho>=0.60 \pm 0.1)$. These correlations indicate that, in addition to the population-level frequency, the individual-level frequency of PMs is conserved. Furthermore, in general, variation between individuals is greater with respect to expression data as compared to genomic data. The one main exception to a high degree of population-level conservation among PMs was that 11 unique polymorphic sites in HB190 were only identified in the genomic dataset from Ghana.

The conservation of the different PMs and their frequencies in the two study populations suggests that these PMs may be evolutionarily conserved over large geographic spaces; thus, associations observed between PMs and pathophysiology in geographically restricted populations, such as the two we examine here in Kenya and Ghana, may be relevant to larger populations, or possibly even globally. We therefore continue by identifying such associations and their relation to previous research findings in the literature. Unique to this study, we explore polymorphisms within HB5 and HB14, which are two common homology blocks present within most var genes. We identified 628 amino-acid PMs within them, 481 (76.6\%) of which are present in both datasets.

\section{Association with severe disease and rosetting}

We used the expression levels of the classic var types, of homology blocks, and of homology blocks polymorphisms to predict severe disease and rosetting. In addition, we evaluate the level of parsitemia both as an independent variable and as an interaction term. Classic var types were defined by the presence/absence of specific motifs in the case of cys2PoLV groups and h3sub var types, and by network analysis in the case of A-like and BS1/CP6 var types, and were obtained from the online supplementary information of (21). The expression levels of classic var types and of homology blocks were used in previous studies to predict severe disease $(13,21)$. A model selection scheme was employed to identify the best logistic model using two initial datasets considering either the expression of classic var gene groups or the expression of homology blocks and their polymorphisms. In both cases, we found that an interaction term with parasitemia was included in the optimal model. 
Predictive ability was measured using an independent subset of the dataset which was randomly selected and was not used in training or data normalization. Prediction of severe disease based on gene expression data was good (AUC $=0.82$ for vars, 0.83 for homology blocks and their polymorphisms) and was not significantly different when considering the two alternative datasets (Fig. 3). However, the use of classic var subtypes included fewer variables namely grpA var genes, the interaction term of the $\mathrm{H} 3$ var subset with parasitemia and parasitemia levels (Table 1). The inclusion of age as a predictor did not improve on the prediction of severe disease.

When predicting rosetting, forecast skill (SS) was considerable for vars ( $\mathrm{SS}=0.41)$ and when prediction was made based on homology blocks and their polymorphisms ( $\mathrm{SS}=0.43$ ) (Fig. 4). As was the case for predicting severe disease, there was no significant difference between the predictive ability of the two models, however the model which included the classic var types had fewer variables. Interestingly, the prediction of rosetting and severe disease included the same two var subsets: grp $A$ and $H 3$, however the rosetting model included $g r p A$ rather than the $H 3$ interaction term with parasitemia (Table 2).

We continued by testing whether specific polymorphisms are associated with the grpA or $H 3$ var subsets (Table 3). Using a similar model selection scheme, we find a set of specific polymorphisms and homology blocks with expression levels correlated with the expression of grpA vars (forecast skill $\mathrm{SS}=0.93, \mathrm{p}=0$ ) (Fig. 5A). To a lesser degree, a different group of polymorphisms is associated with $H 3$ vars $\left(\mathrm{SS}=0.20, \mathrm{p}=10^{-8}\right.$ ) (Fig. $\left.5 \mathrm{~B}\right)$. Based on these results it is likely that a substantial portion of the ability of PMs to predict severe disease relies on their association with grpA-like vars.

\section{Association of var gene polymorphism with age and parasitemia}

In the Warimwe et al. study (45), the overall number of var gene clones declines with age ( $\rho=-0.26$, $\mathrm{p}=0.00019$ ). We tested whether specific homology blocks, or homology block polymorphisms, had changes in their transcription levels in relation to the total number of clones, in a way which go beyond the overall decline with age. We calculated the p-value of the linear regression models (Fig. 6) and corrected for multiple testing as described in (69). We found HB60 to have a moderately lower relative expression rate with increasing age $\left(\rho=-0.30, p=1.2 \cdot 10^{-8}\right.$, FDR $\left.=4.6 \cdot 10^{-5}\right)$ while HB36 displayed an increased relative expression rate with increasing age, however this relationship was not significant $(\rho=0.16, p=0.0017, F D R=0.18)$. HB36 still maintained an overall decline in transcription with age. Similarly, we calculated the association of HB polymorphisms with age. We found that the expression rates of four of HB60s polymorphisms had a similarly strong and declining expression level with host age however none showed a stronger correlation than the expression of the HB itself.

Finally, we tested whether var types and/or var HB polymorphism were associated with parasitemia. Neither the relative expression of var genes nor the relative expression of homology blocks was associated with parasitemia directly. An association between polymorphisms and parasitemia was only detectable in an ensemble of selected logistic models (Pearson's $\rho=0.36, \mathrm{p}=0.02$ ) and not in a consensus model including only stable selected variables.

\section{DISCUSSION}

Antigenic diversity in P. falciparum is a major obstacle for developing vaccines against malaria. The identification of functional and conserved antigenic targets involved in pathogen virulence is greatly needed. The high diversity of the var family has made the identification of reliable var single nucleotide polymorphisms (SNPs) difficult (70). In our study, we limit our analysis to amino-acid level variation 
within homology blocks in the $D B L \alpha$ domain with the aim of identifying reliable variation of functional consequence. We identify conserved amino-acid polymorphisms within the DBL $\alpha$ domain of var genes that are associated with severe pathophysiology and with rosetting. Polymorphisms are a part of multiple conserved and functional protein domain sites and prediction of severe disease based on their transcription level compares in its skill to the prediction of severe disease based on the expression levels of classic var groups. However, amino-acid polymorphisms within homology blocks can uniquely illuminate additional and potentially more direct relationships between protein variation, immunity and function.

We observe the great majority of homology block polymorphisms in both the Kenyan and Ghanaian populations, indicating their evolutionary conservation across large distances of time and space. Furthermore, the fraction of vars with a specific polymorphism was correlated between pairs of individuals sampled from the two populations (mean Spearman's $\langle\rho\rangle=0.60$ ) and within each population $(<\rho>=0.61 \pm 0.1$ in Kenya, $\langle\rho>=0.78 \pm 0.1$ in Ghana). The two study populations are located in very different geographic locations (East versus West Africa), they were sampled more than five years apart, and they represent distinct collection methods (expression versus genomic data). Together, our findings suggest that there is a conserved set of var polymorphisms at the genomic level that is manifest in proportional levels of transcription. It also suggests that variation within homology blocks is conserved and maintained.

The maintenance of homology block polymorphisms across populations in light of the high rates of ectopic recombination known to characterize the var family (71-73), suggests that balancing selection may be at play. Balancing selection may also be responsible for maintaining similar frequencies among variants across populations if certain ratios between alternative PMs at a given site are adaptively optimal for the parasite. Alternatively, conserved frequencies among variants may be attributable to the existence of recombination constraints (or so-called var recombination hierarchies) that orchestrate recombination preferentially among certain var types and/or maintain ratios of certain var types within genomes. They may also be due to shared, recent evolutionary history-i.e. the neutral inheritance and conservation of similar frequencies of variants. The main var groupings reflect both sequence and functional divergence, so it is possible that homology block polymorphisms reflect this functional divergence as well.

Consistent with findings in literature, and with findings based on the same primary data, we found that severe disease correlated with the expression of specific 'classic' var gene types and with higher parasitemia $(21,47,74)$. The optimal model for predicting severe disease that we present here differs from Warimwe et al. in that it includes only group $A$ vars, the interaction term of the $H 3$ subset with parasitemia, and parasitemia levels as an independent variable (Table 1, Fig. 3). In contrast with other studies, we find no association between group B vars and severe disease was identified $(17,44)$. The dependence on the expression levels of vars and on the interaction term with parasitemia may help explain why control of parasitemia by itself is insufficient in preventing severe disease (9).

Interestingly the same var subgroups were also correlated with the rosetting phenotype (Table 2), although this relationship included a different interaction term with parasitemia. This is consistent with the notion of rosetting as a factor contributing to virulence (57). The prediction of rosetting based on the expression of these classic var groups and their interaction with parasitemia explained $40 \%$ of the variance in the validation dataset (Fig. 4). Additional information regarding the host genotype, specifically relating to ABO blood groups may improve prediction of severe disease and of rosetting (36, 57). 
Also consistent with findings in literature, we find an association between certain homology blocks and severe disease, specifically, the transcription of HB60, HB204, HB219, HB367 was associated with severe disease (13). The transcription of HB345, previously associated with a milder symptom gene group, was instead associated with severe disease in the models we describe here. Consistent with previous work, we found that the transcription of HB163 and HB171 was associated with milder symptoms, however in a form including an interaction term with parasitemia. The transcription of HB219, positively associated with severe disease, was negatively associated with severe disease in its interaction with parasitemia.

Adding to the inquiries of previous studies, we also consider variation within homology blocks as predictive variations for disease symptoms. For instance, a polymorphism of HB402: HB402 ${ }_{1 \mathrm{~K}}$, was associated with severe disease in its interaction with parasitemia (Table 1, Fig. 3). The HB402 motif itself was previously associated with severe disease, while not considering individual variation (13). HB5 and HB14 are two common homology blocks present within most var genes, and we identified 628 aminoacid PMs within these two HBs, the majority shared between the two datasets. Thus, our findings suggest that variation within common homology blocks, such as HB5 and HB14, should also be considered when assigning functional, virulence, immunogenic or evolutionary attributes to var types. For example, the transcripts of three variants of HB14 at amino acid position 19 (I, K, M) had either positive or negative associations with severe disease (Table 1).

Since the predictive ability of HBs and HB polymorphisms did not surpass prediction based on 'classic' var genes, we measured the association between the different polymorphisms and the predictive grpA-like and $\mathrm{H} 3$ subset vars. We identify a strong association between the expression of six previously severe implicated HBs (HB60, HB179, HB219, HB367, HB402, HB486) and grpA-like vars (Table 3). In addition, we find novel associations between polymorphisms of HB14 and HB5 and grpA-like vars (Table 3). A weaker association between polymorphisms and the H3 subset was identified and includes: HB219 and its polymorphisms, the polymorphisms of HB5, and HB402 (Table 3). Several HB5 polymorphisms were associated with group A-like and $\mathrm{H} 3$ vars but not with severe disease. Such variation is of interest since it may shed light on the distinction between high and low virulence grpA-like vars (Table 1, Table 3 ).

The expression of group A-like vars is known to be associated with young age and a low degree of acquired immunity (75), which may provide protection against severe disease. An alternative explanation to the pattern is that group A vars may be expressed later during an infection. An expression hierarchy such as this, coupled with higher parasite clearance rates in older individuals, may also explain the phenomenon. However, it does not fit with the presence of chronic asymptomatic infections in adults (76). In our study, HB60 is associated with grpA-like vars and with severe disease, while in previous studies HB36 was shown to be associated with milder symptoms (13). In our analysis, we found that HB60 had moderately but significantly lower relative expression rates in older individuals $\left(\rho=-0.30, p=1.2 \cdot 10^{-8}\right.$, $\operatorname{FDR}=4.6 \cdot 10^{-5}$ ), while HB36 had higher relative expression rates in older individuals $(\rho=0.16, p=0.0017$, $\mathrm{FDR}=0.18$ ) (Fig. 6). Both hypotheses for the correlation with age - an expression hierarchy or specific immunity - could generate the observed patterns, so we view both as plausible in this case.

In the prediction of both severe disease and rosetting based on homology block polymorphisms, the majority of associations we identify are not significant individually from the standpoint of classic logistic or linear regression, but rather provide good predictive ability as an ensemble. Larger sample sizes or a meta-analysis approach may help attain statistical significance at the finer-grain level of detail of 
individual HB polymorphisms. Alternatively, an experimental approach focusing on specific polymorphisms or homology blocks such as HB60 or HB219 would provide more data of this type. Additional limitations to this work include the fact that the dataset sampled from Ghana did not include any var expression level data, and that there was no sampling of asymptomatic individuals in the Kenyan dataset. In addition, the conserved frequency distribution of polymorphisms shared among the study sites may reflect $P$. falciparum populations in Africa, rather than globally. Limited sample size significantly impacted our ability to verify specific associations between polymorphic site variation and pathophysiology.

The association of specific homology blocks and polymorphic sites within homology blocks with severe disease further emphasizes the potential for developing vaccines that could target genes responsible for severe malaria, and thus reduce the burden of mortality and morbidity associated with P. falciparum. Ideally such vaccines will protect young children in Africa against severe disease in wide geographical areas and will not lose their effectiveness through parasite evolution (e.g., antigenic drift). Consistent with previous findings, such putative vaccines could include parasite var gene epitopes involved in severe pathophysiology. Our findings suggest that multiple yet conserved sequence motifs are associated with severe disease, and as such the acquisition of multiple antibodies against them may be sufficient to gain protection. In the case of placental malaria, protective antibodies in pregnant women have been shown to cross-react across geographically diverse placental isolates $(77,78)$. This cross-reactivity has served to motivate the ongoing work on vaccines for pregnant women (77). Targeting variation involving conserved sites within grpA var genes could be of equally great benefit.

ACKNOWLEDGMENTS

We wish to thank the participants, communities and the Ghana Health Service in Bongo District Ghana for their willingness to participate in this study. We would like to thank the field and teams for their technical assistance in the field, as wells the laboratory and research personnel at the Navrongo Health Research Centre for sample collection and parasitological assessment/expertise. Additionally, we would like to thank laboratory personnel at New York University, Noguchi Memorial Institute for Medical Research, and The University of Melbourne for their assistance with laboratory experiments. Finally, we would like to acknowledge Michael Duffy, for his helpful input related to this work. MP is an Investigator at the Howard Hughes Medical Institute.

\section{FINANCIAL SUPPORT}

This research was supported by the Fogarty International Center at National Institutes of Health [Program on the Ecology and Evolution of Infectious Diseases (EEID), Grant number: R01-TW009670]; and the National Institute of Allergy and Infectious Disease, National Institutes of Health [Grant number: R01AI084156]. 


\section{REFERENCES}

1. Greenwood BM, Bradley AK, Greenwood AM, Byass P, Jammeh K, Marsh K, Tulloch S, Oldfield FSJ, Hayes R. 1987. Mortality and morbidity from malaria among children in a rural area of The Gambia, West Africa. Trans R Soc Trop Med Hyg 81:478-486.

2. Okiro EA, Al-Taiar A, Reyburn H, Idro R, Berkley JA, Snow RW. 2009. Age patterns of severe paediatric malaria and their relationship to Plasmodium falciparum transmission intensity. Malar J 8:4.

3. Brewster DR, Kwiatkowski D, White NJ. 1990. Neurological sequelae of cerebral malaria in children. The lancet 336:1039-1043.

4. Greenwood B, Marsh K, Snow R. 1991. Why do some African children develop severe malaria? Parasitol Today 7:277-281.

5. Gupta S, Hill AV, Kwiatkowski D, Greenwood AM, Greenwood BM, Day KP. 1994. Parasite virulence and disease patterns in Plasmodium falciparum malaria. Proc Natl Acad Sci 91:3715-3719.

6. WHO WH. 2016. World malaria report 2016. Geneva WHO 13.

7. Marsh K. 1992. Malaria-a neglected disease? Parasitology 104:S53-S69.

8. Miller LH, Baruch DI, Marsh K, Doumbo OK. 2002. The pathogenic basis of malaria. Nature 415:673-679.

9. Gonçalves BP, Huang C-Y, Morrison R, Holte S, Kabyemela E, Prevots DR, Fried M, Duffy PE. 2014. Parasite Burden and Severity of Malaria in Tanzanian Children. N Engl J Med 370:1799-1808.

10. Smith J, Deitsch KW. 2012. Antigenic variation, adherence, and virulence in malaria. Evol Virulence Eukaryot Microbes 338-361.

11. World Health Organization. 2010. Guidelines for the treatment of malaria2nd ed. World Health Organization, Geneva.

12. Montgomery J, Mphande FA, Berriman M, Pain A, Rogerson SJ, Taylor TE, Molyneux ME, Craig A. 2007. Differential var gene expression in the organs of patients dying of falciparum malaria. Mol Microbiol 65:959-967.

13. Rorick M, Rask T, Baskerville E, Day K, Pascual M. 2013. Homology blocks of Plasmodium falciparum var genes and clinically distinct forms of severe malaria in a local population. BMC Microbiol 13:244.

14. Salanti A, Staalsoe T, Lavstsen T, Jensen AT, Sowa MP, Arnot DE, Hviid L, Theander TG. 2003. Selective upregulation of a single distinctly structured var gene in chondroitin sulphate A-adhering Plasmodium falciparum involved in pregnancy-associated malaria. Mol Microbiol 49:179-191. 
15. Sander AF, Salanti A, Lavstsen T, Nielsen MA, Magistrado P, Lusingu J, Ndam NT, Arnot DE. 2009. Multiple var2csa-Type PfEMP1 Genes Located at Different Chromosomal Loci Occur in Many Plasmodium falciparum Isolates. PLoS ONE 4:e6667.

16. Claessens A, Adams Y, Ghumra A, Lindergard G, Buchan CC, Andisi C, Bull PC, Mok S, Gupta AP, Wang CW. 2012. A subset of group A-like var genes encodes the malaria parasite ligands for binding to human brain endothelial cells. Proc Natl Acad Sci USA 109:E1772-E1781.

17. Kaestli M, Cockburn IA, Cortés A, Baea K, Rowe JA, Beck H-P. 2006. Virulence of malaria is associated with differential expression of Plasmodium falciparum var gene subgroups in a casecontrol study. J Infect Dis 193:1567-1574.

18. Voss TS, Thompson JK, Waterkeyn J, Felger I, Weiss N, Cowman AF, Beck HP. 2000. Genomic distribution and functional characterisation of two distinct and conserved Plasmodium falciparum var gene 5' flanking sequences. Mol Biochem Parasitol 107:103-115.

19. Gardner MJ, Hall N, Fung E, White O, Berriman M, Hyman RW, Carlton JM, Pain A, Nelson KE, Bowman S. 2002. Genome sequence of the human malaria parasite Plasmodium falciparum. Nature 419:498-511.

20. Smith JD. 2014. The role of PfEMP1 adhesion domain classification in Plasmodium falciparum pathogenesis research. Spec Issue 35th Anniv Mol Biochem Parasitol 195:82-87.

21. Warimwe GM, Fegan G, Musyoki JN, Newton CRJC, Opiyo M, Githinji G, Andisi C, Menza F, Kitsao B, Marsh K, Bull PC. 2012. Prognostic Indicators of Life-Threatening Malaria Are Associated with Distinct Parasite Variant Antigen Profiles. Sci Transl Med 4:129ra45-129ra45.

22. Chan J-A, Fowkes FJI, Beeson JG. 2014. Surface antigens of Plasmodium falciparum-infected erythrocytes as immune targets and malaria vaccine candidates. Cell Mol Life Sci 71:3633-3657.

23. Lau CK, Turner L, Jespersen JS, Lowe ED, Petersen B, Wang CW, Petersen JE, Lusingu J, Theander TG, Lavstsen T. 2015. Structural conservation despite huge sequence diversity allows EPCR binding by the PfEMP1 family implicated in severe childhood malaria. Cell Host Microbe 17:118-129.

24. Voss TS, Healer J, Marty AJ, Duffy MF, Thompson JK, Beeson JG, Reeder JC, Crabb BS, Cowman AF. 2006. A var gene promoter controls allelic exclusion of virulence genes in Plasmodium falciparum malaria. Nature 439:1004-1008.

25. Frank M, Deitsch K. 2006. Activation, silencing and mutually exclusive expression within the var gene family of Plasmodium falciparum. Int J Parasitol 36:975-985.

26. Amit-Avraham I, Pozner G, Eshar S, Fastman Y, Kolevzon N, Yavin E, Dzikowski R. 2015. Antisense long noncoding RNAs regulate var gene activation in the malaria parasite Plasmodium falciparum. Proc Natl Acad Sci 112:E982-E991. 
27. Kraemer SM, Kyes SA, Aggarwal G, Springer AL, Nelson SO, Christodoulou Z, Smith LM, Wang W, Levin $\mathrm{E}$, Newbold $\mathrm{Cl}$. 2007. Patterns of gene recombination shape var gene repertoires in Plasmodium falciparum: comparisons of geographically diverse isolates. BMC Genomics 8:45.

28. Baird JK, Barcus MJ, Elyazar IRF, Bangs MJ, Maguire JD, Fryauff DJ, Richie TL, Kalalo W. 2003. Onset of clinical immunity to Plasmodium falciparum among Javanese migrants to Indonesian Papua. Ann Trop Med Parasitol 97:557-564.

29. Gupta S, Snow RW, Donnelly CA, Marsh K, Newbold C. 1999. Immunity to non-cerebral severe malaria is acquired after one or two infections. Nat Med 5:340-343.

30. Chan J-A, Howell KB, Reiling L, Ataide R, Mackintosh CL, Fowkes FJ, Petter M, Chesson JM, Langer C, Warimwe GM. 2012. Targets of antibodies against Plasmodium falciparum-infected erythrocytes in malaria immunity. J Clin Invest 122:3227-3238.

31. Lusingu JP, Jensen AT, Vestergaard LS, Minja DT, Dalgaard MB, Gesase S, Mmbando BP, Kitua AY, Lemnge MM, Cavanagh D. 2006. Levels of plasma immunoglobulin $G$ with specificity against the cysteine-rich interdomain regions of a semiconserved Plasmodium falciparum erythrocyte membrane protein 1, VAR4, predict protection against malarial anemia and febrile episodes. Infect Immun 74:2867-2875.

32. Nielsen MA, Staalsoe T, Kurtzhals JA, Goka BQ, Dodoo D, Alifrangis M, Theander TG, Akanmori BD, Hviid L. 2002. Plasmodium falciparum variant surface antigen expression varies between isolates causing severe and nonsevere malaria and is modified by acquired immunity. J Immunol 168:3444-3450.

33. Angeletti D, Albrecht L, Blomqvist K, Quintana Mdel P, Akhter T, Bachle SM, Sawyer A, Sandalova T, Achour A, Wahlgren M. 2012. Plasmodium falciparum rosetting epitopes converge in the SD3-loop of PfEMP1-DBL1alpha. PLoS One 7:e50758.

34. Guillotte $M$, Juillerat A, Igonet $S$, Hessel A, Petres S, Crublet E, Le Scanf C, Lewit-Bentley A, Bentley GA, Vigan-Womas I, Mercereau-Puijalon O. 2015. Immunogenicity of the Plasmodium falciparum PfEMP1-VarO Adhesin: Induction of Surface-Reactive and Rosette-Disrupting Antibodies to VarO Infected Erythrocytes. PLOS ONE 10:e0134292.

35. Albrecht L, Moll K, Blomqvist K, Normark J, Chen Q, Wahlgren M. 2011. var gene transcription and PfEMP1 expression in the rosetting and cytoadhesive plasmodium falciparum clone FCR3S1.2. Malar J 10:17.

36. Barragan A, Kremsner PG, Wahlgren M, Carlson J. 2000. Blood Group A Antigen Is a Coreceptor inPlasmodium falciparum Rosetting. Infect Immun 68:2971-2975.

37. Juillerat A, Lewit-Bentley A, Guillotte M, Gangnard S, Hessel A, Baron B, Vigan-Womas I, England P, Mercereau-Puijalon O, Bentley GA. 2011. Structure of a plasmodium falciparum PfEMP1 rosetting 
domain reveals a role for the $\mathrm{N}$-terminal segment in heparin-mediated rosette inhibition. Proc Natl Acad Sci USA 108:5243-5248.

38. Kraemer SM, Smith JD. 2006. A family affair: var genes, PfEMP1 binding, and malaria disease. Curr Opin Microbiol 9:374-380.

39. Lavstsen T, Turner L, Saguti F, Magistrado P, Rask TS, Jespersen JS, Wang CW, Berger SS, Baraka V, Marquard AM. 2012. Plasmodium falciparum erythrocyte membrane protein 1 domain cassettes 8 and 13 are associated with severe malaria in children. Proc Natl Acad Sci USA 109:E1791-E1800.

40. Rowe JA, Moulds JM, Newbold Cl, Miller LH. 1997. P. falciparum rosetting mediated by a parasitevariant erythrocyte membrane protein and complement-receptor 1. Nature 388:292-295.

41. Smith JD, Subramanian G, Gamain B, Baruch DI, Miller LH. 2000. Classification of adhesive domains in the plasmodium falciparum erythrocyte membrane protein 1 family. Mol Biochem Parasitol 110:293-310.

42. Kirchgatter K, Portillo H del A. 2002. Association of severe noncerebral Plasmodium falciparum malaria in Brazil with expressed PfEMP1 DBL1 alpha sequences lacking cysteine residues. Mol Med 8:16.

43. Kyriacou HM, Stone GN, Challis RJ, Raza A, Lyke KE, Thera MA, Kone AK, Doumbo OK, Plowe CV, Rowe JA. 2006. Differential var gene transcription in Plasmodium falciparum isolates from patients with cerebral malaria compared to hyperparasitaemia. Mol Biochem Parasit 150:211-218.

44. Rottmann M, Lavstsen T, Mugasa JP, Kaestli M, Jensen AT, Müller D, Theander T, Beck H-P. 2006. Differential expression of var gene groups is associated with morbidity caused by Plasmodium falciparum infection in Tanzanian children. Infect Immun 74:3904-3911.

45. Warimwe GM, Keane TM, Fegan G, Musyoki JN, Newton CR, Pain A, Berriman M, Marsh K, Bull PC. 2009. Plasmodium falciparum var gene expression is modified by host immunity. Proc Natl Acad Sci 106:21801-21806.

46. Turner L, Lavstsen T, Berger SS, Wang CW, Petersen JEV, Avril M, Brazier AJ, Freeth J, Jespersen JS, Nielsen MA, Magistrado P, Lusingu J, Smith JD, Higgins MK, Theander TG. 2013. Severe malaria is associated with parasite binding to endothelial protein $C$ receptor. Nature 498:502-505.

47. Normark J, Nilsson D, Ribacke U, Winter G, Moll K, Wheelock CE, Bayarugaba J, Kironde F, Egwang TG, Chen Q. 2007. PfEMP1-DBL1alpha Amino acid motifs in severe disease states of plasmodium falciparum malaria. Proc Natl Acad Sci USA 104:15835-15840.

48. Abdi Al, Muthui M, Kiragu E, Bull PC. 2014. Measuring Soluble ICAM-1 in African Populations. PloS One 9:e108956. 
49. Salanti A, Dahlbäck M, Turner L, Nielsen MA, Barfod L, Magistrado P, Jensen AT, Lavstsen T, Ofori MF, Marsh K. 2004. Evidence for the involvement of VAR2CSA in pregnancy-associated malaria. J Exp Med 200:1197-1203.

50. Robinson BA, Welch TL, Smith JD. 2003. Widespread functional specialization of Plasmodium falciparum erythrocyte membrane protein 1 family members to bind CD36 analysed across a parasite genome. Mol Microbiol 47:1265-1278.

51. Cockburn IA, Mackinnon MJ, O'Donnell A, Allen SJ, Moulds JM, Baisor M, Bockarie M, Reeder JC, Rowe JA. 2004. A human complement receptor 1 polymorphism that reduces Plasmodium falciparum rosetting confers protection against severe malaria. Proc Natl Acad Sci U S A 101:272277.

52. Louicharoen C, Patin E, Paul R, Nuchprayoon I, Witoonpanich B, Peerapittayamongkol C, Casademont I, Sura T, Laird NM, Singhasivanon P, Quintana-Murci L, Sakuntabhai A. 2009. Positively selected G6PD-mahidol mutation reduces Plasmodium vivax density in Southeast Asians. Science 326:1546-1549.

53. Mangano VD, Modiano D. 2014. An evolutionary perspective of how infection drives human genome diversity: the case of malaria. Immunogenetics Transplant Spec Sect Eff Endog Immune Stimul 30:39-47.

54. May J, Evans JA, Timmann C, Ehmen C, Busch W, Thye T, Agbenyega T, Horstmann RD. 2007. Hemoglobin variants and disease manifestations in severe falciparum malaria. J Am Med Assoc 297:2220-2226.

55. Modiano D, Bancone G, Ciminelli BM, Pompei F, Blot I, Simporé J, Modiano G. 2008. Haemoglobin $S$ and haemoglobin C: "Quick but costly" versus "slow but gratis" genetic adaptations to Plasmodium falciparum malaria. Hum Mol Genet 17:789-799.

56. Zimmerman PA, Ferreira MU, Howes RE, Mercereau-Puijalon O. 2013. Red Blood Cell Polymorphism and Susceptibility to Plasmodium vivax.

57. Rowe JA, Handel IG, Thera MA, Deans A-M, Lyke KE, Koné A, Diallo DA, Raza A, Kai O, Marsh K, others. 2007. Blood group $O$ protects against severe Plasmodium falciparum malaria through the mechanism of reduced rosetting. Proc Natl Acad Sci 104:17471-17476.

58. Hedrick PW. 2011. Population genetics of malaria resistance in humans. Heredity 107:283-304.

59. Taylor HM, Kyes SA, Harris D, Kriek N, Newbold Cl. 2000. A study of var gene transcription in vitro using universal var gene primers. Mol Biochem Parasitol 105:13-23.

60. Kraemer SM, Smith JD. 2003. Evidence for the importance of genetic structuring to the structural and functional specialization of the Plasmodium falciparum var gene family. Mol Microbiol 50:1527-1538. 
61. Lavstsen T, Salanti A, Jensen AT, Arnot DE, Theander TG. 2003. Sub-grouping of Plasmodium falciparum 3D7 var genes based on sequence analysis of coding and non-coding regions. Malar J 2:27.

62. Barry AE, Leliwa-Sytek A, Tavul L, Imrie H, Migot-Nabias F, Brown SM, McVean GAV, Day KP. 2007. Population Genomics of the Immune Evasion (var) Genes of Plasmodium falciparum. PLoS Pathog 3:e34.

63. Rice P, Longden I, Bleasby A. 2000. EMBOSS: the European molecular biology open software suite. Trends Genet 16:276-277.

64. Goujon M, McWilliam H, Li W, Valentin F, Squizzato S, Paern J, Lopez R. 2010. A new bioinformatics analysis tools framework at EMBL-EBI. Nucleic Acids Res 38:W695-W699.

65. Rask TS, Hansen DA, Theander TG, Gorm Pedersen A, Lavstsen T. 2010. Plasmodium falciparum erythrocyte membrane protein 1 diversity in seven genomes-divide and conquer. PLoS Comput Biol 6:e1000933.

66. Simon N, Friedman J, Hastie T, Tibshirani R. 2013. A sparse-group lasso. J Comput Graph Stat 22:231-245.

67. Dabney A, Storey JD, Warnes G. 2004. Q-value estimation for false discovery rate control. Medicine (Baltimore) 344:539-548.

68. Benjamini Y, Hochberg Y. 1995. Controlling the false discovery rate: a practical and powerful approach to multiple testing. J R Stat Soc Ser B Methodol 289-300.

69. Storey JD. 2002. A direct approach to false discovery rates. J R Stat Soc Ser B Stat Methodol 64:479-498.

70. Manske M, Miotto O, Campino S, Auburn S, Almagro-Garcia J, Maslen G, O’Brien J, Djimde A, Doumbo O, Zongo I. 2012. Analysis of Plasmodium falciparum diversity in natural infections by deep sequencing. Nature 487:375-379.

71. Claessens A, Hamilton WL, Kekre M, Otto TD, Faizullabhoy A, Rayner JC, Kwiatkowski D. 2014. Generation of antigenic diversity in Plasmodium falciparum by structured rearrangement of Var genes during mitosis. PLoS Genet 10:e1004812.

72. Freitas-Junior LH, Bottius E, Pirrit LA, Deitsch KW, Scheidig C, Guinet F, Nehrbass U, Wellems TE, Scherf A. 2000. Frequent ectopic recombination of virulence factor genes in telomeric chromosome clusters of P. falciparum. Nature 407:1018-1022.

73. Kirkman LA, Lawrence EA, Deitsch KW. 2014. Malaria parasites utilize both homologous recombination and alternative end joining pathways to maintain genome integrity. Nucleic Acids Res 42:370-379. 
74. Jensen AT, Magistrado P, Sharp S, Joergensen L, Lavstsen T, Chiucchiuini A, Salanti A, Vestergaard LS, Lusingu JP, Hermsen R. 2004. Plasmodium falciparum associated with severe childhood malaria preferentially expresses PfEMP1 encoded by group A var genes. J Exp Med 199:1179-1190.

75. Cham GK, Turner L, Lusingu J, Vestergaard L, Mmbando BP, Kurtis JD, Jensen AT, Salanti A, Lavstsen T, Theander TG. 2009. Sequential, ordered acquisition of antibodies to Plasmodium falciparum erythrocyte membrane protein 1 domains. J Immunol 183:3356-3363.

76. Dal-Bianco MP, Köster KB, Kombila UD, Kun JF, Grobusch MP, Ngoma GM, Matsiegui PB, Supan C, Salazar CLO, Missinou MA. 2007. High prevalence of asymptomatic Plasmodium falciparum infection in Gabonese adults. Am J Trop Med Hyg 77:939-942.

77. Bockhorst J, Lu F, Janes JH, Keebler J, Gamain B, Awadalla P, Su X, Samudrala R, Jojic N, Smith JD. 2007. Structural polymorphism and diversifying selection on the pregnancy malaria vaccine candidate VAR2CSA. Mol Biochem Parasitol 155:103-112.

78. Staalsoe T., Shulman C.E., Bulmer J.N., Kawuondo K., Marsh K., Hviid L. 2004. Variant surface antigen-specific IgG and protection against clinical consequences of pregnancy-associated Plasmodium falciparum malaria. Lancet 363:283-289. 


\section{TABLES}

Table 1. Statistics for logistic regression models predicting severe disease

\begin{tabular}{|c|c|c|}
\hline model data & stable predictive variables & AUC \\
\hline $\begin{array}{l}\text { HBs, PMs, } \\
\text { interaction terms } \\
\text { with parasite } \\
\text { density (pdt) }\end{array}$ & 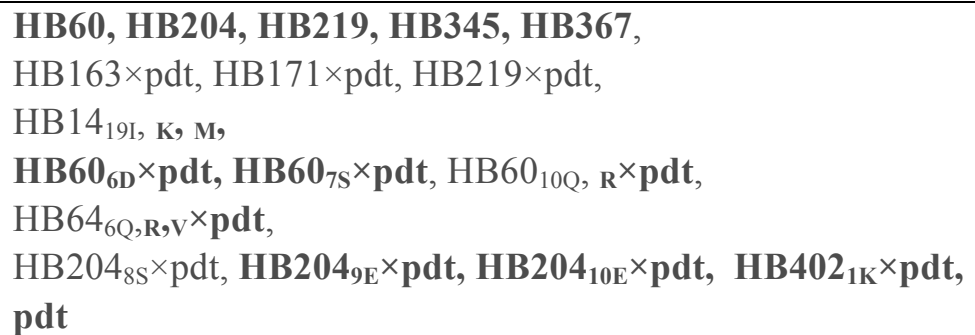 & 0.83 \\
\hline $\begin{array}{l}\text { classic var genes and } \\
\text { interaction with } \\
\text { parasite density }\end{array}$ & grpA, H3×pdt, pdt & 0.82 \\
\hline
\end{tabular}

positive effect independent variables are shown in boldface

subscripts indicate the position and a polymorphism and the amino-acid varient 
Table 2. Statistics for linear regression models predicting rosetting

\begin{tabular}{|c|c|c|}
\hline model data & stable predictive variables & SS \\
\hline $\begin{array}{l}\text { HBs, PMs, } \\
\text { interaction terms } \\
\text { with parasite } \\
\text { density (pdt) }\end{array}$ & 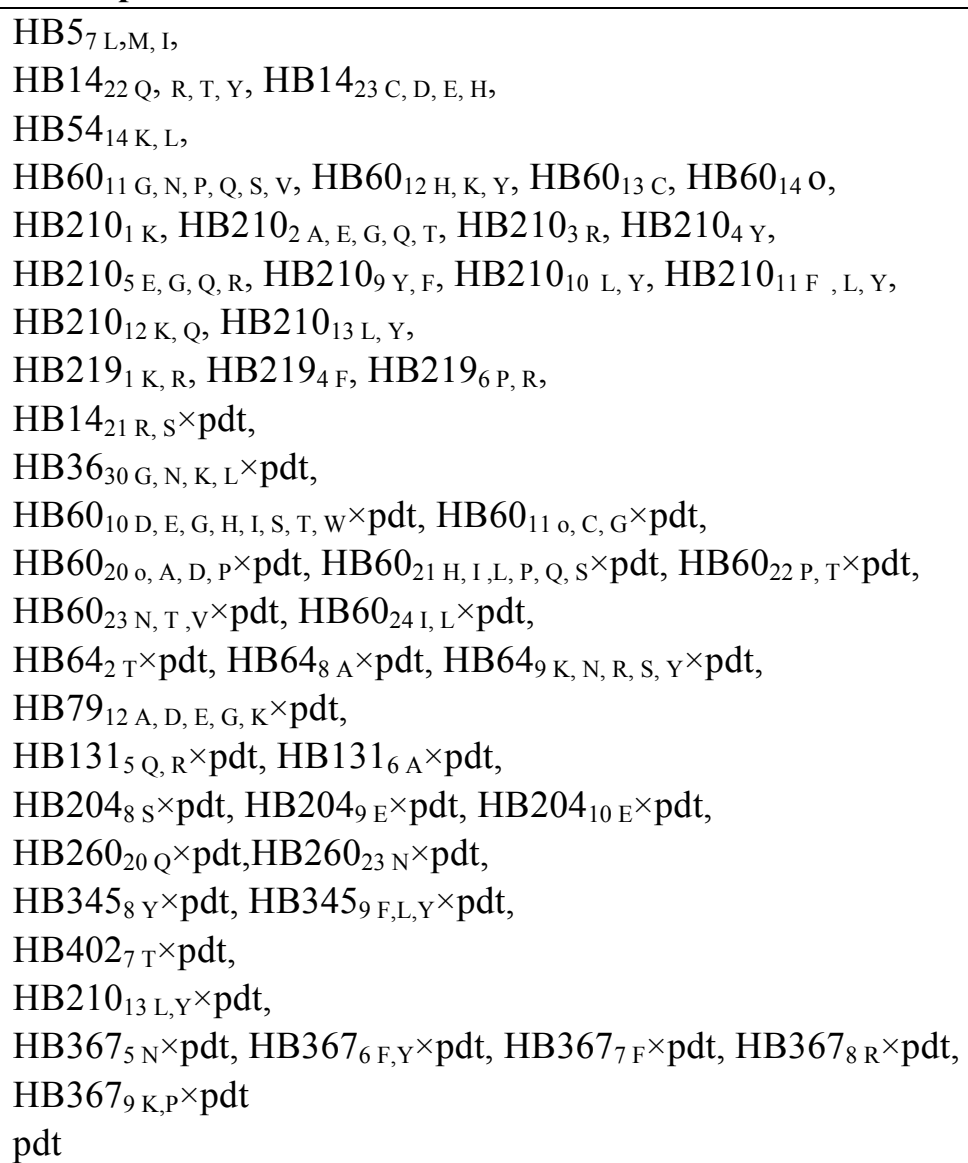 & 0.4 \\
\hline $\begin{array}{l}\text { classic var genes and } \\
\text { interaction with } \\
\text { parasite density } \\
\text { (pdt) }\end{array}$ & $\operatorname{grpA}, \mathrm{H} 3$, grpA $\times$ pdt, pdt & 0.4 \\
\hline
\end{tabular}

positive effect independent variables are shown in boldface subscripts indicate the position and a polymorphism and the amino-acid variant 
Table 3. Statistics for logistic regression models association with grpA and $H 3$ var subsets

\begin{tabular}{|c|c|c|}
\hline predicting transcription of & stable predictive variables & SS \\
\hline $\operatorname{grp} A$ & $\begin{array}{l}\text { HB60, HB179, HB219, HB367, HB402, HB486, } \\
\text { HB5 }_{20 S}, \mathrm{HB14}{ }_{1 \mathrm{~V}}, \mathrm{HB}_{4} 4_{4, \mathrm{~A}, \mathrm{~N}}\end{array}$ & 0.93 \\
\hline$H 3$ & 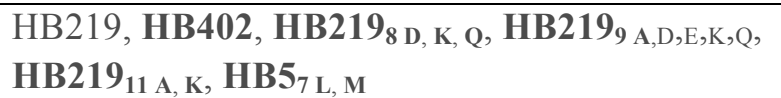 & 0.20 \\
\hline
\end{tabular}

positive effect independent variables are shown in boldface

subscripts indicate the position and a polymorphism and the amino-acid varient 


\section{FIGURES}

A

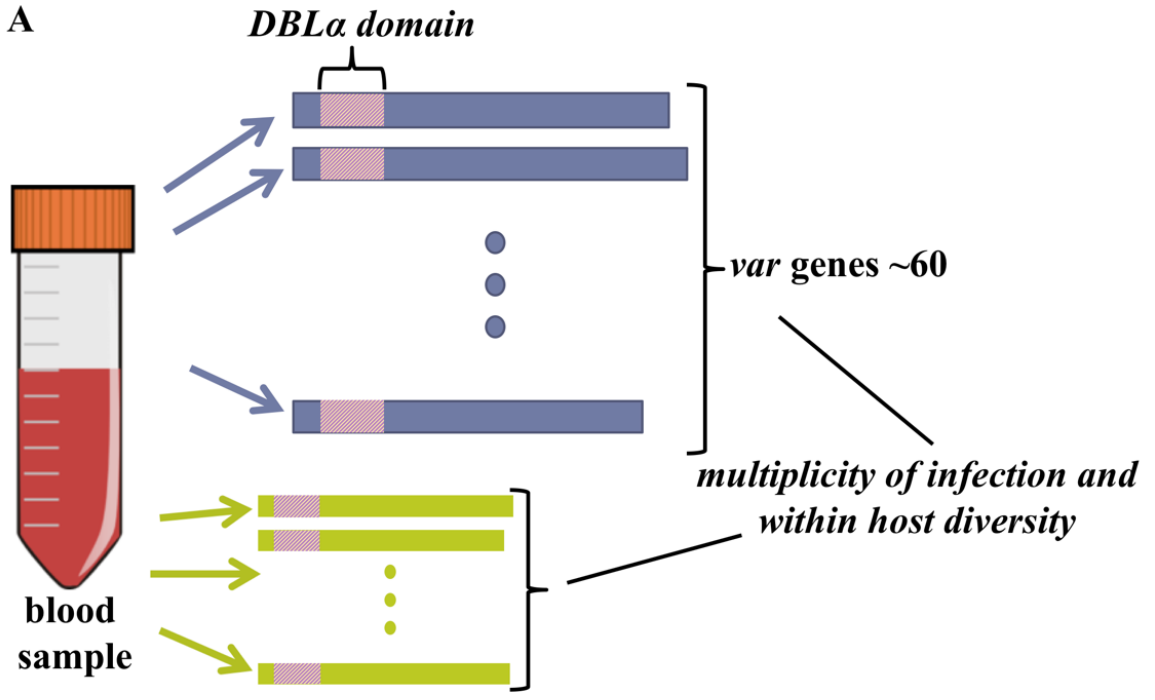

B

NTS DBL $1 \alpha$ CIDR $1 \alpha$ DBL $2 \delta$ CIDR2 $\beta$ TM ATS

$\begin{aligned} \mathrm{NTS} \text { DBL } \alpha \text { CIDR } 1 \alpha 1 \mathrm{DBL} 2 \beta & \mathrm{C} 2 \mathrm{DBL} 3 \gamma \quad \mathrm{DBL} 2 \delta \text { CIDR } 2 \beta \mathrm{TM} \text { ATS } \\ & \bullet \text { within host var multiplicity } \\ & \bullet \text { and architectural type } \\ & \bullet \text { variation }\end{aligned}$

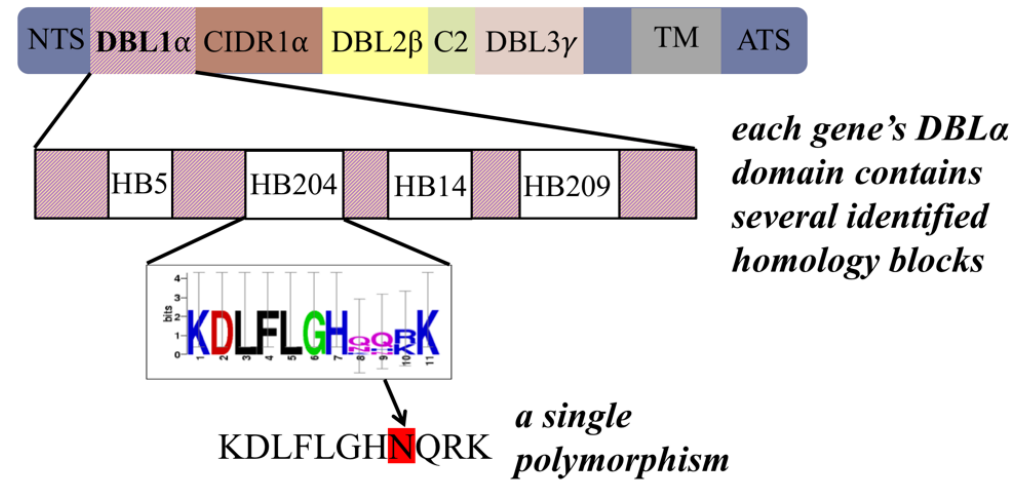

Figure 1. Amino-acid polymorphisms of PfEMP1 A. Each Plasmodium falciparum parasite contains within its genome a set of approximately 60 var genes. A set of var genes, representing one or more infections, is sequenced from each blood sample, taken from individuals participating in the pilot study. The DBLa domain, located in the head domain of most var genes is sequenced and further analyzed. B. PfEMP1 proteins can be classified into multiple architectural types with an alternative domain structure, three of which are depicted for illustration; N-terminal segment (NTS), DBL, CIDR, C2 domain, transmembrane (TM), acidic terminal segment (ATS) (38). The DBLa domain is further analyzed for the identification of short alignable sequences termed homology blocks. Sequences that compose homology blocks contain specific amino-acid motifs, but are not exact copies of each other. Variation, or single amino acid polymorphisms, within homology blocks is used in further analyses and is tested for association with disease clinical manifestation. 
fraction of individuals with a.a. polymorphism

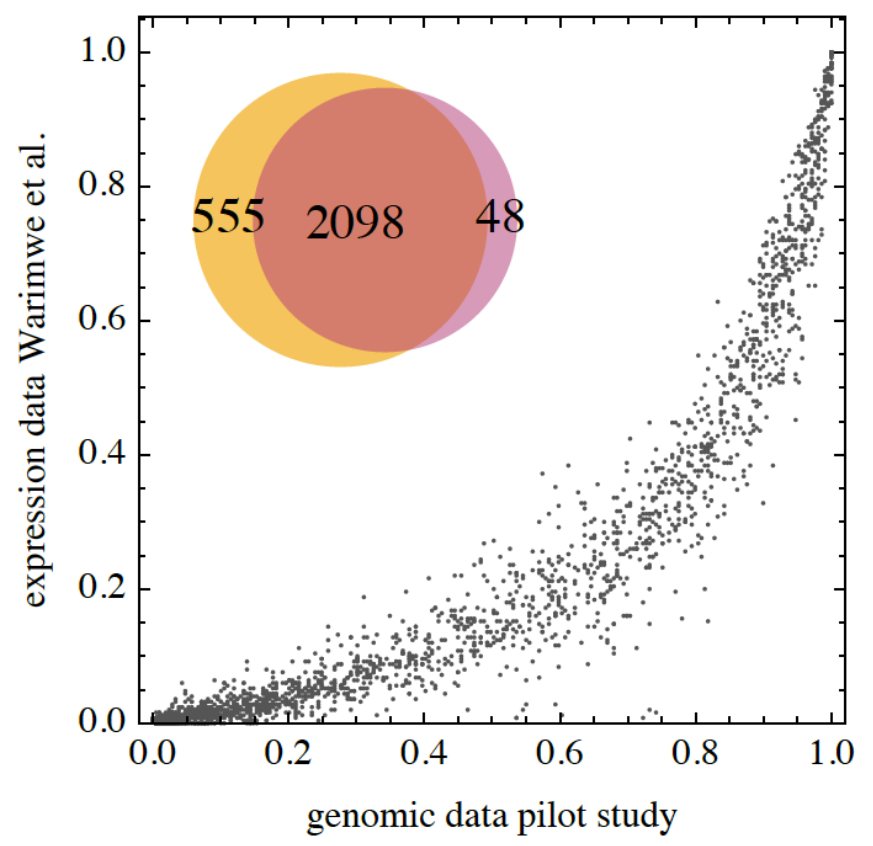

Figure 2. Frequency of amino-acid polymorphisms in the two study populations The fraction of vars in which single amino-acid polymorphisms within homology blocks are present in the study population in Ghana, and in expression data from the Warimwe et al. study. A total of 2701 amino acid polymorphisms were identified in either genomic sequences in the Ghana study (left) or expression data in Kenya (right). 2098 amino-acid polymorphisms were shared between the two study populations, 555 were unique to genomic data in the Ghana study, and 48 were unique to the expression data in the Kenya study. 


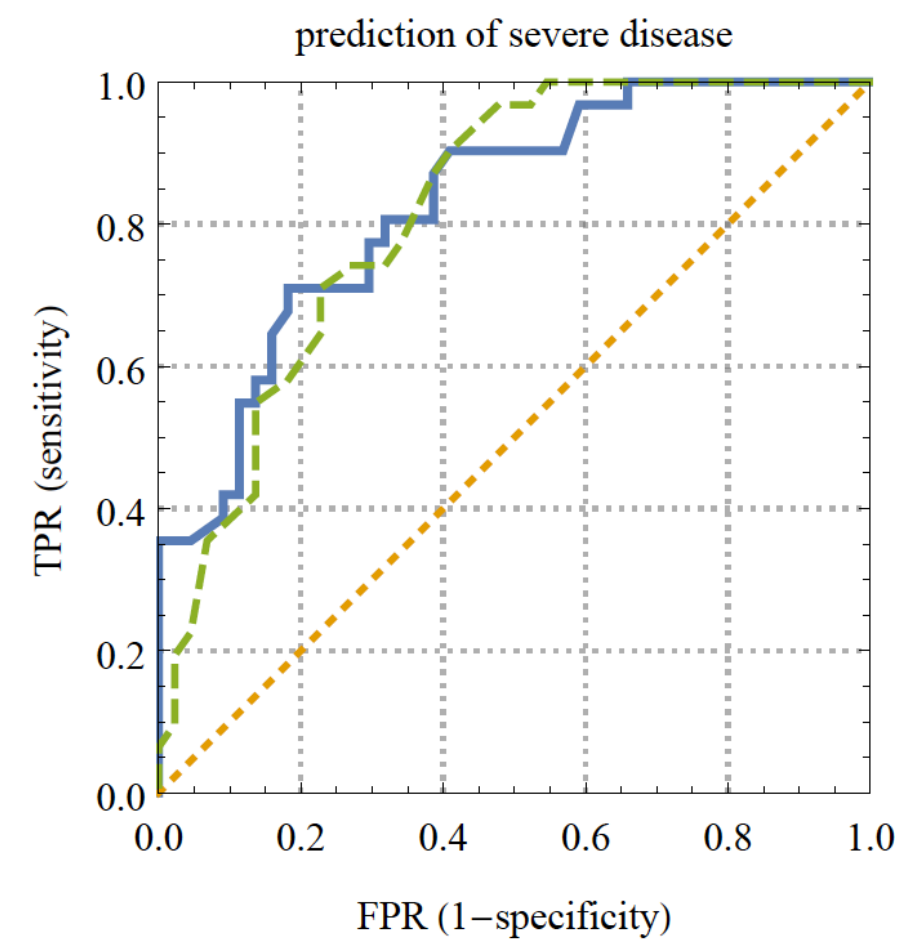

Figure 3. ROC curve for prediction of severe disease in an out of bag dataset The area under the curve represents the probability that a randomly chosen subject with severe disease is correctly rates or ranked with greater suspicion than a randomly chosen individual without severe disease. sold line = prediction based on amino acid polymorphisms, dashed line = prediction based on "classic" var gene groups. Scores were calculated using a logistic regression model, and ROC curves represent prediction in an out of bag dataset of 75 individuals not used in the normalization of expression levels or in the training of the model. 


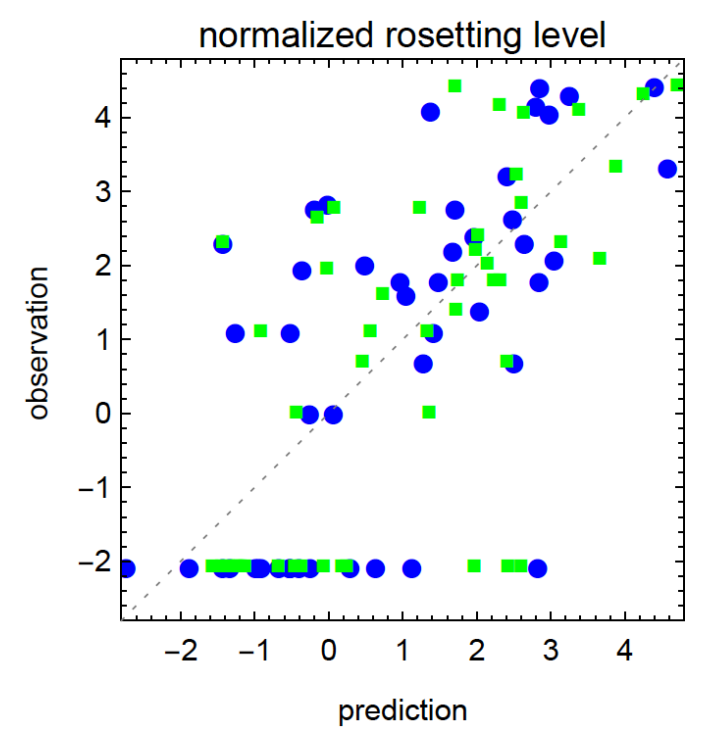

Figure 4. Prediction of rosetting Prediction of normalized rosetting levels using a linear model and the transcription levels of var genes or of HBs and their amino acid polymorphisms. Prediction is shown for an out of bag dataset of 75 individuals not used in the normalization of expression levels or in the training of the model. $\bullet=$ prediction based on amino acid polymorphisms, $\square=$ prediction based on "classic" var gene groups. 
$\mathbf{A}$

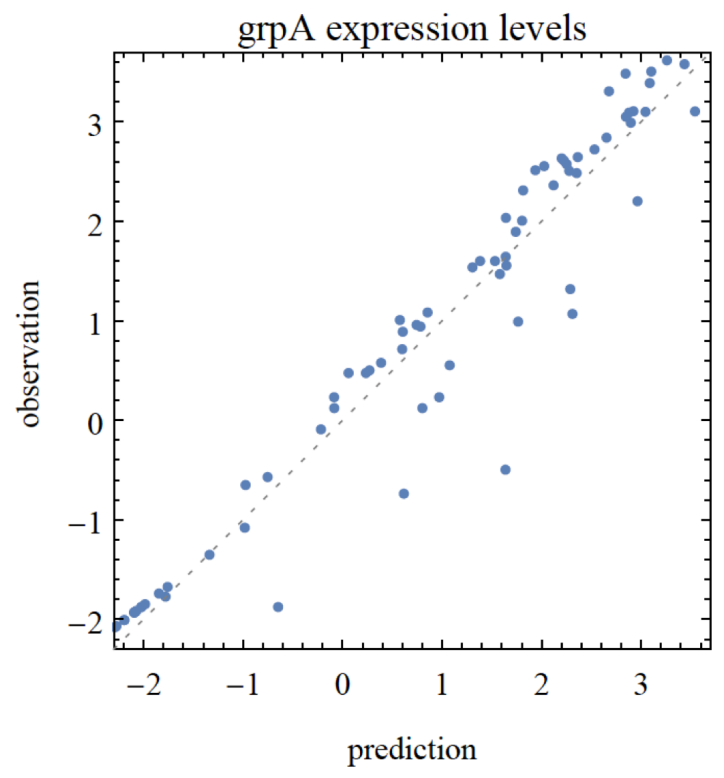

B

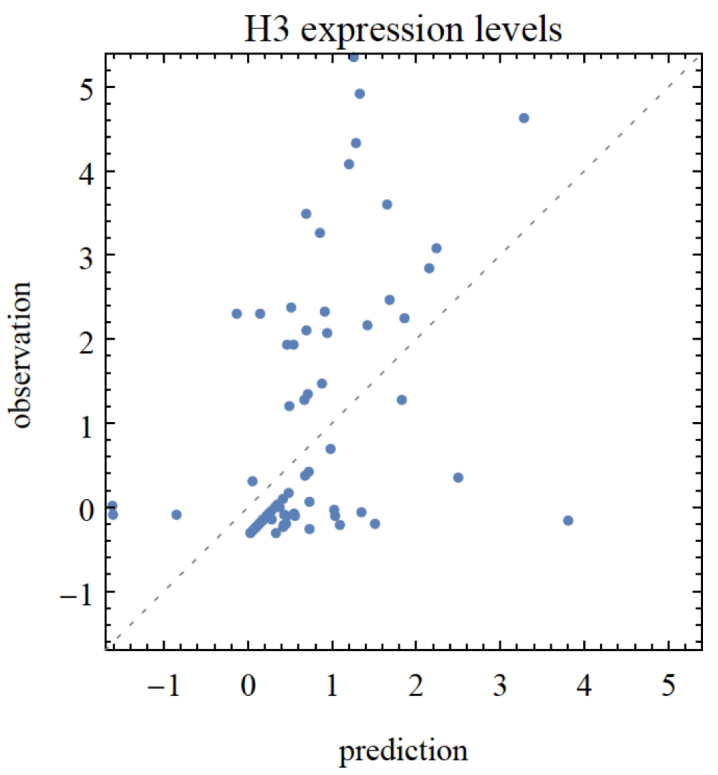

Figure 5. Association between group A like and $\mathrm{H3}$ vars, homology blocks and their polymorphisms Prediction is shown for an out of bag dataset of 75 individuals not used in the normalization of expression levels or in the training of the model. A Prediction of the transcription levels of group A like vars using a linear model and the transcription levels of HBs and their amino acid polymorphisms. B Prediction of the transcription levels of $\mathrm{H3}$ vars using a linear model and the transcription levels of HBs and their amino acid polymorphisms. 

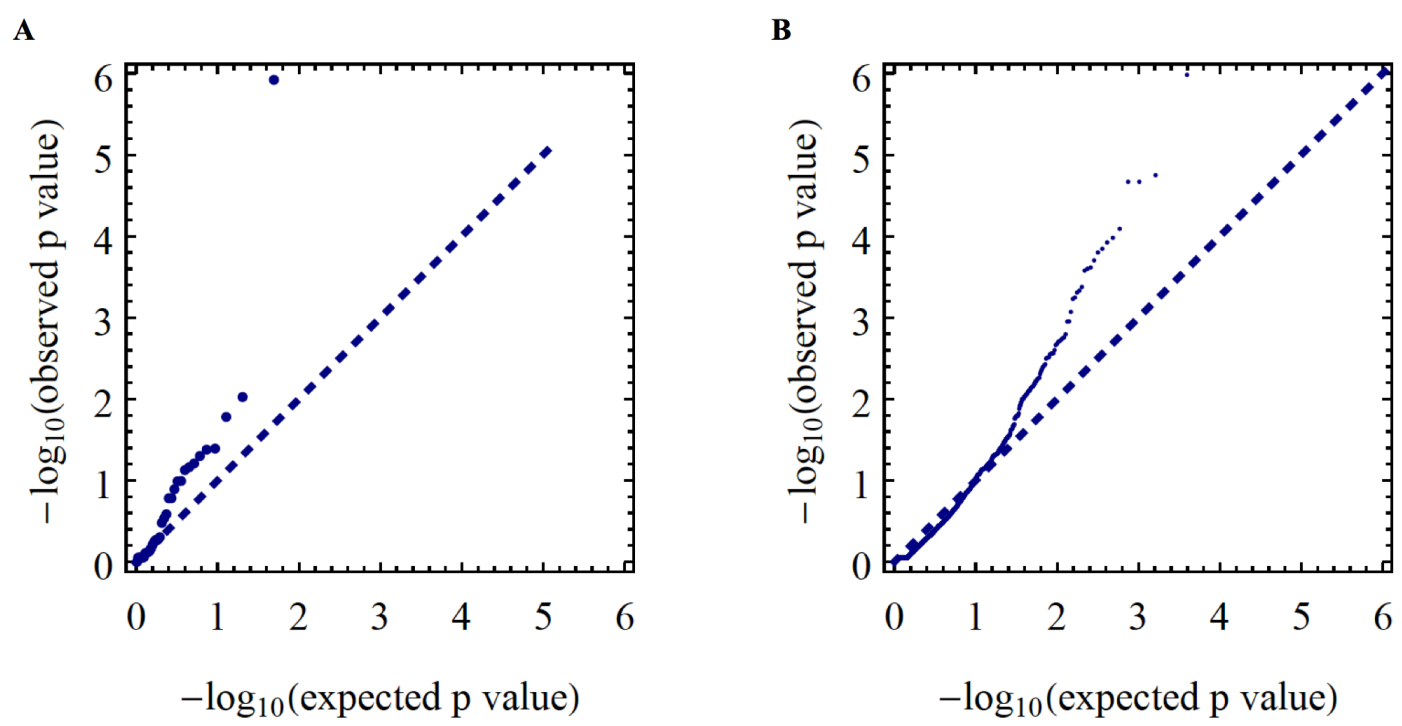

Figure 6. Q-Q plot for association between $\mathrm{HB}$ and $\mathrm{HB}$ polymorphisms with age The quantilequantile (Q-Q) plot showing the significance of linear models associating age with the relative expression of HBs (A) or of HB polymorphisms (B). 